\title{
IV. Complexity induced anisotropic bimodal intermittent turbulence in space plasmas
}

Tom Chang and Sunny W.Y. Tam

Center for Space Research, Massachusetts Institute of Technology, Cambridge, Massachusetts 02139 USA

Cheng-chin Wu

Department of Physics and Astronomy, University of California, Los Angeles, California 90095 USA

\begin{abstract}
The "physics of complexity" in space plasmas is the central theme of this exposition. It is demonstrated that the sporadic and localized interactions of magnetic coherent structures arising from the plasma resonances can be the source for the coexistence of nonpropagating spatiotemporal fluctuations and propagating modes. Non-Gaussian probability distribution functions of the intermittent fluctuations from direct numerical simulations are obtained and discussed. Power spectra and local intermittency measures using the wavelet analyses are presented to display the spottiness of the small-scale turbulent fluctuations and the non-uniformity of coarse-grained dissipation that can lead to magnetic topological reconfigurations. The technique of the dynamic renormalization group is applied to the study of the scaling properties of such type of multiscale fluctuations. Charged particle interactions with both the propagating and nonpropagating portions of the intermittent turbulence are also described.
\end{abstract}




\section{INTRODUCTION}

Simultaneous coexistence of propagating modes and intermittent nonlinear spatiotemporal structures is the norm of the state of the plasma medium in the space environment. The "physics" of the bimodal state of such type of admixture of turbulent fluctuations may be understood from the point of view of the development and interactions of coherent structures arising from plasma resonance sites. ${ }^{1-6}$

In this treatise, we shall consider the dynamical complexity in space plasmas from such a concept. Results of two-dimensional direct numerical simulations ${ }^{7-9}$ including the calculated fluctuation probability distribution functions and local intermittency measures based on the wavelet transforms will be presented to characterize the sporadic, localized, and scale-dependent nature of the intermittent turbulence.

The concepts of scale invariance and symmetry-breaking phenomena of such invariance properties will be described based on the dynamical theory of the renormalization group. ${ }^{10}$ Illustrative examples will be provided to elucidate the utility of this powerful theoretical technique in addressing the complexity of space plasmas.

The intricate interactions among the charged particles in the plasma medium and the propagating and nonpropagating intermittent fluctuations will be considered and an example related to the energization of the auroral ions will be provided. ${ }^{11}$

We have endeavored to make this exposition sufficiently self-contained. Approximately one third of this treatise is a review of previous work and the remaining two thirds are discussions of new research results. 


\section{THE ORIGIN OF COMPLEXITY}

\section{A. Alfvénic resonances and coherent structures}

Most field theoretical discussions begin with the concept of propagation of waves. For example, in the MHD formulation, one can combine the basic equations and express them in the following propagation forms:

$$
\rho d \mathbf{V} / d t=\mathbf{B} \cdot \nabla \mathbf{B}+\cdots, \quad d \mathbf{B} / d t=\mathbf{B} \cdot \nabla \mathbf{V}+\cdots
$$

where the ellipses represent the effects of the anisotropic pressure tensor, the compressible and dissipative effects, and all notations are standard. Equations (1) admit the well-known Alfvén waves. For such waves to propagate the propagation vector $\mathbf{k}$ must contain a field-aligned component, i.e., $\mathbf{B} \cdot \nabla \rightarrow i \mathbf{k} \cdot \mathbf{B} \neq 0$. However, at sites where the parallel component of the propagation vector vanishes (i.e., at the resonance sites), the fluctuations are localized. Around these resonance sites (usually in the form of curves), it may be shown that the fluctuations are held back by the background magnetic field, forming Alfvénic coherent structures. ${ }^{1-4,12,13}$

\section{B. Coarse-grained helicity}

Let us now consider the geometry of the Alfvénic coherent structures. For an ideal MHD system, it has been suggested by Taylor ${ }^{14}$ that in a relaxed state such a structure would be approximately force-free (i.e., $\mathbf{J} \times \mathbf{B}=0$ ) due to the approximate conservation of the coarsegrained helicity defined as $K=\int \mathbf{A} \cdot \mathbf{B} d V$ integrated over the coherent structure, where $\mathbf{J}$ and $\mathbf{B}$ are the current density and magnetic field and $\mathbf{A}$ is the vector potential.

To obtain some physical insight of these structures, let us consider the special situation for the auroral region and/or the solar wind and make the reasonable assumption that the perturbed magnetic field fluctuations are much smaller than and essentially transverse to the mean magnetic field $B_{0}$ (which will be temporarily assumed to be uniform for the current discussion). Thus, let us write $\mathbf{B}=\left(\delta B_{x}, \delta B_{y}, B_{0}\right)$, where $\mathrm{z}$ is in the direction of the mean magnetic field, and $(\mathrm{x}, \mathrm{y})$ are orthogonal coordinates normal to $\mathrm{z}$. The force-free condition for constant $B_{0}$ and $\nabla \cdot \mathbf{J}=0$ then 
leads approximately to the scalar condition $\mathbf{B} \cdot \nabla J_{z}=0$, obtained by taking the z-component of the curl of $\mathbf{J} \times \mathbf{B}=0 . .^{11,15,16}$ It can be shown that, with the inclusion of the kinetic effects through the anisotropic pressure terms and the generalized Ohm's law, the above results are still approximately valid. We have, then, approximately,

$$
B_{0} \partial J_{z} / \partial z=-\left(\delta B_{x} \partial / \partial x+\delta B_{y} \partial / \partial y\right) J_{z}+\cdots
$$

where the ellipsis represents the other modifying effects. For convenience, let us introduce the flux function $\psi$ by writing $(\partial \psi / \partial y,-\partial \psi / \partial x)=\left(\delta B_{x}, \delta B_{y}\right)$ for the perturbed transverse components of the magnetic field in the $(x, y)$ directions such that $\nabla \cdot \mathbf{B}=0$ is satisfied. Then, $J_{z}$ and $\psi$ are governed by Eq. (2) and the Ampere's law (neglecting the modifying effects represented by the ellipsis).

A simple example of the flux function and axial current density satisfying the above conditions would be the class of circularly cylindrical solutions of $\psi(r)$ and $J_{z}(r)$. Generally, the solutions would be more involved because of the variabilities of the local conditions of the plasma and the three-dimensional geometry. Moreover, the dynamic coherent structures with the inclusion of plasma pressure and other modifying effects (including electron-inertia terms) would be even more complicated. However, we expect these structures to be usually in the form of field-aligned flux tubes, Fig. 1.

Generally, there exist various types of propagation modes (whistler modes, electromagnetic ion cyclotron waves, etc.) in magnetized plasmas. Thus, we envision a corresponding number of different types of plasma resonances and associated coherent structures that typically characterize the dynamics of the plasma medium under the influence of a background magnetic field.

Generally, such coherent structures may take on the shapes of convective forms, nonlinear solitary structures, pseudo-equilibrium configurations, as well as other types of spatiotemporal varieties. These structures might be locally generated or convected from elsewhere (such as some 
of the observed structures in the solar wind that might have been originated from the Sun's surface $^{17 a}$. Some of these structures may be more stable than the others. They, however, generally are not purely laminar entities as they are composed of bundled fluctuations of all frequencies. Because of the nature of the physics of complexity, it will be futile to attempt to evaluate and/or study the details and stabilities of each of these infinite varieties of structures; although some basic understanding of each type of these structures will generally be helpful in the comprehension of the full complexity of the underlying nonlinear plasma dynamics.

These coherent structures will wiggle, migrate, deform and undergo different types of motions and interactions under the influence of the local plasma and magnetic topologies. In the next section, we will consider how the coherent structures can interact and produce the type of intermittency generally observed in a complex dynamical plasma.

\section{Interactions and complexity}

When coherent magnetic flux tubes of the same polarity migrate toward each other, strong local magnetic shears are created, Fig. 2. It has been demonstrated by $\mathrm{Wu}$ and $\mathrm{Chang}^{7-9}$ that existing sporadic nonpropagating fluctuations will generally migrate toward the strong local shear region. Eventually the mean local energies of the coherent structures will be dissipated into these concentrated fluctuations in the coarse-grained sense and induce reconfigurations of the magnetic field geometry. Figure 3(a) displays 2D MHD simulation results for homogeneous turbulence. The calculations were carried out with $(512 \times 512)$ grid points in a doubly periodic $(x . y)$ domain of length $2 \pi$ in both directions. The left panel of Fig. 3(a) gives contours of the magnetic flux at $t=300$. For reference, the sound wave and Alfvén wave traveling times through a distance $2 \pi$ are about 4.4 and 60, respectively. Regions of intense current density (strong magnetic shear) are evident in the right panel of Fig. 3(a). (We found the results are very similar with or without a zcomponent magnetic field.) Figure 3(b) is essentially the same as Fig. 3(a) except that these results were obtained with $(1024 \times 1024)$ grid points. Thus, our simulation results are quite 
robust. To understand the effect of the coarse-grained dissipation, we have performed 2D MHD simulations of $(512 \times 512)$ grid points with a sheared magnetic field of $B_{x}=0.1 \cos y$. Figure 3(c) are the contours of the magnetic flux and current density distributions for the shear case. The neutral sheet regions are near $y=0.5 \pi, 1.5 \pi$. The clustering of the coherent structures (enhanced coarse-grained dissipation) and the reconfiguration of the contours of the magnetic fields near the neutral sheets are evident in the displays.

Such enhanced intermittency at the intersection regions has been observed by Bruno et al. ${ }^{17,18}$ in the solar wind using the tools of wavelet analyses and local intermittency measure (LIM). As mentioned above, the coarse-grained dissipation will then initiate "fluctuation-induced nonlinear instabilities";, ${ }^{419}$ and, thereby reconfigure the topologies of the coherent structures of the same polarity into a combined lower local energetic state, eventually allowing the coherent structures to merge locally. On the other hand, when coherent structures of opposite polarities approach each other due to the forcing of the surrounding plasma, they might repel each other, scatter, or induce magnetically quiescent localized regions. Under any of the conditions of the above interaction scenarios, new fluctuations will be generated. And, these new fluctuations can provide new resonance sites; thereby nucleating new coherent structures of varied sizes.

All such interactions can occur at any location of a flux tube along its field-aligned direction, and the phenomenon. is fully three-dimensional. In order to gain some insight of the physical picture of the overall dynamics of the interactions of the coherent structures, let us again consider the auroral zone or the solar wind as an illustrative example. We make the plausible assumption that some aspects of the plasma dynamics may be approximately understood in terms of the formulation of reduced magnetohydrodynamics (RMHD).$^{20,21}$ In this approximation, we assume that the mean magnetic field is much larger than the transverse fields, and the field-aligned fluctuations of the magnetic and velocity components are much smaller than their transverse 
counterparts. As a consequence, the density of the plasma is uniform. Writing the equations in SI units with $\rho=1$ and $\mu_{0}=1$, we have: 22,23

$$
d \psi / d t=B_{z} \partial \phi / \partial z, \quad d \omega / d t=\mathbf{B} \cdot \nabla j
$$

where $\psi(x, y)$ is the transverse flux function defined by $\mathbf{B}=\mathbf{e}_{z} \times \nabla \psi+B_{z} \mathbf{e}_{z}, \phi(x, y)$ is the transverse stream function defined by $\mathbf{v}_{\perp}=\mathbf{e}_{z} \times \nabla \phi$, and $\omega=\nabla_{\perp}^{2} \phi$ is the vorticity, $j=\nabla_{\perp}^{2} \psi$ is the field-aligned current density with $d / d t=\partial / \partial t+v \cdot \nabla$. The equations are written in the moving frame along the mean magnetic field direction $z$, and $(x, y)$ are the transverse orthogonal directions.

From Eqs. (3), we note that the primary nonlinear interactions occur generally in the transverse direction to the mean magnetic field. And, the coupling in the field-aligned direction is essentially linear. Thus, fluctuations generated by the transverse nonlinear interactions will scatter and evolve nonlinearly primarily in the transverse direction. At this point, we realize that the RMHD formulation is too restrictive as some of the interactions of the flux tubes may become more oblique and thereby allowing the fluctuations to attain a broader range of values of $k_{\|}$than otherwise would have been admitted by the RMHD approximation. Thus, a significant amount of the fluctuations generated by the interactions can become commensurate with the plasma dispersion relation and propagate in the field-aligned direction as Alfvén waves due to this threedimensional complexity-induced enhanced transport. Eventually a dynamic topology of a complex state of coexisting propagating and nonpropagating magnetic fluctuations is created. In the auroral region, the plasma may be electron-inertia dominated and the above discussion can be easily generalized to include such kinetic effects.

\section{SCALING AND SYMMETRY BREAKING}

\section{A. Path integral and the dynamic renormalization group}

In the above sections, we provided some convincing arguments as well as numerical and observational evidences indicating that space plasma turbulence is generally in a state of 
topological complexity. By "complex" topological states we mean magnetic topologies that are not immediately deducible from the elemental (e.g., MHD and/or Vlasov) equations. ${ }^{24}$ Below, we shall briefly address the salient features of the analogy between topological and equilibrium phase transitions. A thorough discussion of these ideas may be found in Chang ${ }^{4,11,25}$ (and references contained therein).

For nonlinear stochastic systems exhibiting complexity, the correlations among the fluctuations of the random dynamical fields are generally extremely long-ranged and there exist many correlation scales. The dynamics of such systems are notoriously difficult to handle either analytically or numerically. On the other hand, since the correlations are extremely long-ranged, it is reasonable to expect that the system will exhibit some sort of invariance under coarsegraining scale transformations. A powerful technique that utilizes this invariance property is the method of the dynamic renormalization group..$^{10,25,26}$ The technique is a generalization of the static renormalization group introduced by Wilson. ${ }^{27}$

As it has been demonstrated by Chang et al., ${ }^{26}$ based on the path integral formalism, the behavior of a nonlinear stochastic system far from equilibrium may be described in terms of a "stochastic Lagrangian $L$ ", such that the probability density functional $P$ of the stochastic system is expressible as:

$$
P(\varphi(\mathbf{x}, t))=\int D[\chi] \exp \left\{-i \int L(\dot{\varphi}, \varphi, \chi) d x d t\right\}
$$

where $\varphi(\mathbf{x}, t)=\phi_{i}(i=1,2, \ldots, N)$ are the stochastic variables such as the fluctuating magnetic, velocity and electric fields, and $\chi(\mathbf{x}, t)=\chi_{i}(i=1,2, \ldots, N)$ are the conjugate stochastic momentum variables that may be rigorously derived from the underlying stochastic equations governing $\varphi \cdot 10,25,26$

Then, the renormalization-group (coarse-graining) transformation may be formally expressed as:

$$
\partial L / \partial \ell=R L
$$


where $R$ is the renormalization-group (coarse-graining) transformation operator and $\ell$ is the coarse-graining parameter for the continuous group of transformations. It will be convenient to consider the state of the stochastic Lagrangian in terms of its parameters $\left\{P_{n}\right\}$. Equation (5), then, specifies how the Lagrangian, $L$, flows (changes) with $\ell$ in the affine space spanned by $\left\{P_{n}\right\}$, Fig. 4 .

\section{B. Forced and/or self-organized criticality}

Generally, there exists. a number of fixed points (singular points) in the flow field, at which $d L / d \ell=0$. At each such fixed point ( $L^{*}$ or $L^{* *}$ in Fig. 4 ), the correlation length should not be changing. However, the renormalization-group transformation requires that all length scales must change under the coarse-graining procedure. Therefore, to satisfy both requirements, the correlation length must be either infinite or zero. When it is at infinity, the dynamical system is then at a state of forced and/or self-organized criticality (FSOC), ${ }^{25,28}$ analogous to the state of criticality in equilibrium phase transitions. ${ }^{29}$ To study the stochastic behavior of a nonlinear dynamical system near such a dynamical critical state (e.g., the one characterized by the fixed point $L^{*}$ ), we linearize the renormalization-group operator $R$ about $L^{*}$. The mathematical consequence of this approximation is that, close to dynamic criticality, certain linear combinations of the parameters that characterize the stochastic Lagrangian $L$ will correlate with each other in the form of power laws. These include, in particular, the $(k, \omega)$, i.e. mode number and frequency, spectra of the correlations of the various fluctuations of the dynamic field variables.

Such power law behavior has been detected in the probability distributions of solar flare intensities, ${ }^{30}$ in the $\mathrm{AE}$ burst occurrences as a function of the $\mathrm{AE}$ burst strength, ${ }^{31}$ in the global auroral UVI imagery of the statistics of size and energy dissipated by the magnetospheric system, ${ }^{32}$ in the probability distributions of spatiotemporal magnetospheric disturbances as seen in the UVI images of the nighttime ionosphere, ${ }^{33}$ and in the probability distributions of durations 
of Bursty Bulk Flows; ${ }^{34}$ although some of the above interpretations of observed data may also be amenable to alternative explanations. ${ }^{35}$

In addition, it can be demonstrated from such a linearized analysis of the dynamic renormalization group that generally only a small number of (relevant) parameters are needed to characterize the stochastic state of the system near criticality ${ }^{25}$ justifying the recent work suggesting that certain dynamic characteristics of the magnetotail could be modeled by the deterministic chaos of low-dimensional nonlinear systems. ${ }^{36-38}$

\section{Illustrative examples}

The intermittency description for plasma turbulence of nonpropagating fluctuations may be modeled by the combination of a localized chaotic functional growth equation of a set of relevant order parameters and a functional transport equation of the control parameters. ${ }^{6,39}$ Below, we shall provide two simple phenomenological models, which may have some relevance. to the auroral zone or the solar wind. ${ }^{s}$

\section{Model I}

Assuming that the parallel mean magnetic field $B_{0}$ is sufficiently strong and the magnetic fluctuations dominate in the transverse directions, we introduce the flux function $\psi$ for the transverse fluctuations as follows,

$$
\mathbf{B}=\mathbf{e}_{z} \times \nabla \psi+B_{0} \mathbf{e}_{z}
$$

where we have set $B_{z}=B_{0}=$ constant. The coherent structures for such a system are generally flux tubes approximately aligned in the mean parallel direction. ${ }^{11}$ Conservation of helicity (e.g., under the RMHD approximation) indicates that the integral of $\psi$ over a flux tube is approximately constant. Instead of invoking the RMHD formalism, however, here we simply consider $\psi$ as an order parameter. As the flux tubes merge and interact, they may correlate over long distances, which, in turn, will induce long relaxation times near FSOC.,25 Let us assume that the transverse size of the system is sufficiently broad compared to the cross sections of the 
coherent structures (or flux tubes), such that we may invoke homogeneity and assume the dynamics to be independent of boundary effects. We may then model the dynamics of flux tube mergings and interactions, in the crudest approximation, in terms of the following order-disorder intermittency equation:

$$
\partial \psi_{k} / \partial t=-\Gamma_{k} \partial F / \partial \psi_{-k}+f_{k}
$$

where $\psi_{k}$ are the Fourier components of the flux function, $\Gamma_{k}$ an analytic function of $k^{2}$, $F\left(\psi_{k}, k\right)$ the state function, and $f_{k}$ a random noise which includes all the other effects that are not included in the first two terms of this crude model.

\section{Model II}

In the above model, we have neglected both the effects of diffusion and convection. We next construct a phenomenological model that includes the transport of cross-field diffusion. We now assume the state function to depend on the flux function $\psi$ and the local pseudo-energy measure $\xi$. Thus, in addition to the dynamic equation (7), we now also include a diffusion equation for $\xi$. In Fourier space, we have

$$
\partial \xi_{k} / \partial t=-D k^{2} \partial F / \partial \xi_{-k}+h_{k}
$$

where $\xi_{k}$ are the Fourier components of $\xi, D(k)$ is the diffusion coefficient, and the state function is now $F\left(\psi_{k}, \xi_{k}, k\right)$, and $h_{k}$ is a random noise. By doing so, we separate the slow pseudo-energy transport due to diffusion of the local pseudo-energy measure $\xi$ from the noise term of (7). We note that an approach similar to these ideas have been considered by Klimas $e t$ al. $^{40}$

\section{Dynamic renormalization-group analysis}

We have performed renormalization-group analyses as outlined above for the two kinetic models described above. We note that under the dynamic renormalization-group (DRG) transformation, the correlation function $C$ of $\psi_{k}$ should scale as: 


$$
e^{a_{c} \ell} C(k, \omega)=C\left(k e^{\ell}, \omega e^{a_{\omega} \ell}\right)
$$

where $\omega$ is the Fourier transform of $t, \ell$ the renormalization parameter as defined in Sec. IIIA, and $\left(a_{c}, a_{\omega}\right)$ the correlation and dynamic exponents. Thus, $C / \omega^{a_{c} / a_{\omega}}$ is an absolute invariant under the DRG, or $C \sim \omega^{-\lambda}$, where $\lambda=-a_{c} / a_{\omega}$. DRG analysis of Model I with Gaussian noise yields the value of $\lambda$ to be approximately equal to 2.0 . DRG analyses performed for Model II for Gaussian noises for several approximations yield the value for $\lambda$ to be approximately equal to 1.88 to 1.66

Interestingly, for both models, DRG calculations give an approximate value of -1.0 for the $\omega$-exponent for the trace of the transverse magnetic correlation tensor. Matthaeus and Goldstein ${ }^{41}$ had suggested that such an exponent might represent the superposition of discrete structures emerged from the solar convection zone. This value for the $\omega$-exponent is meanfield-like, thus is probably universally applicable to the low frequency fluctuations such as those considered by $M \& G$ as well as other more intermittent small scale fluctuations observed in space plasmas. Also, the corresponding $k$-exponent is found to be approximately equal to -2 for both models. These results compare rather favorably with the results of our $2 \mathrm{D}$ MHD numerical simulations, Fig. 5.

\section{Symmetry breaking}

As the dynamical system evolves in time (autonomously or under external forcing), the state of the system (i.e., the values of the set of the parameters characterizing the stochastic Lagrangian, $L$ ) changes accordingly. A number of dynamical scenarios are possible. For example, the system may evolve from a critical state A (characterized by $L^{* *}$ ) to another critical state B (characterized by $L^{*}$ ) as shown in Fig. 4 . In this case, the system may evolve continuously from one critical state to another. On the other hand, the evolution from the critical state $A$ to critical state $C$ as shown in Fig. 4 would probably involve a dynamical instability characterized by a first-order-like topological phase transition (fluctuation-induced nonlinear 
instability) because the dynamical path of evolution of the stochastic system would have to cross over a couple of topological (renormalization-group) separatrices. For such a situation the underlying magnetic topology and its related plasma state will generally undergo drastic changes. Similar ideas along these lines have been advanced by Sitnov et $a l^{42}$ and simulated based on the cellular automata calculations of sandpile models. ${ }^{43,44}$

Under either of these above scenarios, the spectra indices will generally change either continuously or abruptly. Such type of multifractal phenomena is commonly observed in the magnetotail, the auroral zone, and the solar wind. ${ }^{11,17,18,45-49}$

Alternatively, a dynamical system may evolve from a critical state $A$ to a state $D$ (as shown in Fig. 4) which may not be situated in a regime dominated by any of the fixed points; in such a case, the final state of the system will no longer exhibit any of the characteristic properties that are associated with dynamic criticality. As another possibility, the dynamical system may deviate only moderately from the domain of a critical state characterized by a particular fixed point such that the system may still display low-dimensional scaling laws, but the scaling laws may now be deduced from straightforward dimensional arguments. The system is then in a so-called meanfield state. (For general references of symmetry breaking and nonlinear crossover, see Chang and Stanley ${ }^{50}$; Chang et al. ${ }^{51,52}$; Nicoll et al. ${ }^{53,54}$ )

Experimental observations of plasma fluctuations in the space plasma environment generally yield broken power law spectra similar to those displayed in Fig. 5 of the $2 \mathrm{D}$ numerical simulation results. Such abrupt changes of scaling powers of the $k$-spectra are signatures of symmetry breaking. The broken symmetries may be due to the abrupt change of the degree of intermittency of fluctuations from large to small scales, or due to the change of the underlying physics (e.g., from MHD to kinetic processes), or variations of external forcing, or finite boundaries and other effects. 


\section{DYNAMICAL INTERMITTENCY}

Nearly all fluctuations in space plasmas exhibit intermittency. For turbulent dynamical systems with intermittency, the transfer of energy (or other relevant scalars and tensors) due to fluctuations from one scale to another deviates significantly from uniformity. A technique of measuring the degree of intermittency is the study of the departure from Gaussianity the probability distribution functions of turbulent fluctuations at different scales. To demonstrate this point, let us refer to the 2D numerical simulation results described in Sec. II. For example, we may generate the probability distribution function $P\left(\delta B^{2}, \delta\right)$ of $\delta B^{2}(x, \delta)$ $\equiv B^{2}(x+\delta)-B^{2}(x)$ at a given time $t$ for such simulations, where $\delta$ is the scale of separation in the $\mathrm{x}$-direction. The left panel of Fig. 6(a) displays the calculated results of $P\left(\delta B^{2}, \delta\right)$ from the $(512 \times 512) 2 \mathrm{D}$ simulation for the homogeneous case for several scales $\delta$.

From this figure, we note that the deviation from Gaussianity becomes more and more pronounced at smaller and smaller scales. In an interesting paper by Hnat et $a l^{55}$, they demonstrated that such probability distributions for solar wind fluctuations exhibit approximate mono-power scaling according to the following functional relation:

$$
P\left(\delta B^{2}, \delta\right)=\delta^{-s} P_{s}\left(\delta B^{2} \delta^{-s}, \delta\right)
$$

where $s$ is the mono-scaling power. We found that mono-power scaling also holds approximately for our simulated results with the value of $s$ equal to approximately 0.335 as shown in the right panel of Fig. 6(a).

The reason for mono-power scaling for $\delta B^{2}$ may be understood in terms of the renormalization-group arguments presented in Sec. III. If we assume that $\delta B^{2}$ is one of the relevant eigenoperators near a critical fixed point, then the probability distribution function for $P\left(\delta B^{2}, \delta\right), \delta B^{2}$, as well as $\delta$ will scale linearly as follows:

$$
P^{\prime}=P \exp \left(a_{p} l\right), \quad \delta B^{2}{ }^{\prime}=\delta B^{2} \exp \left(a_{B^{2}} l\right), \quad \delta^{\prime}=\delta \exp \left(a_{\delta} l\right)
$$


where $\left(a_{p}, a_{B^{2}}, a_{\delta}\right)$ are scaling powers. Thus, we obtain two irreducible absolute invariants: $P / \delta^{a_{P} / a_{\delta}}$ and $\delta B^{2} / \delta^{a_{B^{2}} / a_{\delta}}$. Since $P=P\left(\delta B^{2}, \delta\right)$, there must be a functional relation between these two invariants. ${ }^{51,52}$ Therefore, we obtain the following scaling relation among $\left(P, \delta B^{2}, \delta\right): P / \delta^{a_{P} / a_{\delta}}=F\left(\delta B^{2} / \delta^{a_{B^{2}} / a_{\delta}}\right)$.

Without loss of generality, we may choose $a_{\delta}=1$. With the additional constraint that the probability distribution functions are normalized, we immediately obtain the expression of Hnat et al. as shown in Eq. (10).

Actually the scaling relation (10) is approximate in that the tails of the scaled distributions in the right panel of Fig. 6(a) do not exactly fall onto one curve. This is the intrinsic nature of the strong intermittency at small scales. Thus, representations of the probability density functions will involve multi-parameters in general and may sometimes be represented more accurately, for example, by the Castaing or Kappa distributions ${ }^{56-59}$ Their scaling properties are more subtle and will not be considered in this treatise.

As it has been pointed out in Sec. IIC, sporadic nonpropagating fluctuations will generally migrate toward regions of strong shear. We have calculated the probability distribution functions for regions near and away from the neutral sheet for the $(512 \times 512) 2 \mathrm{D}$ simulation for the shear case, Figs. $6(b, c)$. As expected, the degree of intermittency (deviation from Gaussianity) is much stronger near the neutral sheet than away from it. Both regions exhibit approximate mono-power scaling; though, again the tail regions do not scale exactly, particularly near the neutral sheet.

Since the degree of intermittency generally increases inversely with scale, it will be interesting to study the degrees of intermittency locally at different scales. This can be accomplished by the method of Local Intermittency Measure (LIM) using the wavelet transforms. A wavelet transform generally is composed of modes which are square integrable localized functions that are capable of unfolding fluctuating fields into space and scale. ${ }^{60}$ Left panel of Fig. $7(1)$ is the 
power spectrum of a complex Morlet wavelet transform of the current density $J_{z}$ for the (512x512) 2D homogeneous case. We notice that the intensity of the current density is sporadic and varies nonuniformly with scale.

We now define $\operatorname{LIM}(1)$ as the ratio of the squared wavelet amplitude $|\psi(x, \delta)|^{2}$ and its space averaged value $\left\langle|\psi(x, \delta)|^{2}\right\rangle_{x}$. We note that $\operatorname{LIM}(1)=1$ for the Fourier spectrum. To emphasize the variation of intensity with scale, we also consider the logarithm of LIM(1). It has been suggested by Meneveau ${ }^{61}$ that the space average of the square of LIM(1), which is a scale dependent measure of the kurtosis or flatness, is a convenient gauge of the deviation of intermittency from Gaussianity. We denote this measure by LIM(2). It is equal to 3 if the probability distribution is Gaussian. Bottom right panel of Fig. 7(1) and Fig. 8(a) are graphical displays of the calculated results of $\log \operatorname{LIM}(1)$ and $\operatorname{LIM}(2)$ for the $(512 \times 512) 2 \mathrm{D}$ simulation for the homogeneous case using the complex Morlet transform. We notice that the fluctuations are indeed scale dependent, localized and strongly intermittent at small scales. Similar experimental results using the wavelet transforms have been found, for example, by Consolini $e t$ al ${ }^{62}$ for the magnetotail and Bruno et al..$^{17}$ for the solar wind.

Figure $7(2)$ gives graphical displays of $\log L I M(1)$ for the $(512 \times 512) 2 D$ simulation for the shear case for both away from and at the neutral sheet region. We note that the degree of intermittency is indeed much stronger at the neutral sheet region. This result is also confirmed by the graphical displays of LIM(2) in Fig. 8(b).

In the above, we considered some simplified models and numerical examples that may have some relevance to intermittent turbulence in space plasmas. Realistic models for these phenomena will generally be much more complicated. For example, from the RMHD formulation of (3), we recognize that there should at least be two competing order parameters. These are the flux function $\psi$ and the stream function $\phi$ (which is linearly proportional to the electrostatic potential). Thus, the intermittency equation (such as (7)) needs to be generalized to 
accommodate these coupled order parameters. Within the RMHD formulation, there exist useful Hamiltonian and operator-algebra structures, ${ }^{63}$ which should prove invaluable in developing the generalized state function $F$ of the coupled order parameters and the state variables as well as the intermittency equation itself. Formulations of coupled order parameters and their related theoretical analyses for a variety of criticality problems in condensed matter physics have been considered by Chang et al. ${ }^{10}$ (and references contained therein).

In addition, the transport equation such as Eq. (8) for the global system should generally also include convection and acceleration terms in addition to that of diffusion. ${ }^{6}$ Thus, at the minimum our model transport equation must take on the form of the RMHD (3) with the addition of "coarse-grained" dissipation terms which generally will be functionals of the coupled order

parameters. It should also contain terms representing the complexity-induced enhanced fieldaligned transport. These generalizations will not be considered in this treatise.

\section{ENERGIZATION OF IONS BY INTERMITTENT FLUCTUATIONS IN THE}

\section{AURORAL ZONE}

It has long been recognized that the commonly observed broadband, low frequency electric field fluctuations are responsible for the acceleration of oxygen ions in the auroral zone: In order for the fluctuating electric field to resonantly accelerate the ions continuously as the ions evolve upward along the field lines, they must be in continuous resonance with the ions. There did not seem to exist a fully viable mechanism that can generate a spectrum of fluctuations broadband and incoherent enough to fulfill this stringent requirement.

Assuming that the RMHD formulation holds approximately in the auroral zone, the electrostatic fluctuations transverse to the field-aligned direction are given approximately by the velocity fluctuations: $\mathbf{v} \times B_{z} \mathbf{e}_{2}$. The ordering due to the stream function $\phi$ may be important in the auroral zone and therefore, the electrostatic fluctuations can be quite significant there. Because of the small scales involved, the dynamic intermittency produced by the merging and 
interactions of the coherent structures is probably generated by the whistler turbulence, electroninertia related tearing modes, and/or other collisionless modes. ${ }^{11}$ Therefore, a significant portion of the fluctuations would be kinetic. Nevertheless, the electric field fluctuations would still be predominantly transverse and electrostatic. Thus, a significant portion of the low frequency fluctuations commonly observed in the auroral zone are probably contributed by these nonpropagating intermittent fluctuations.

Below, we shall briefly discuss how such fluctuations can efficiently energize the oxygen ions from ionospheric to magnetospheric energies. Assuming the oxygen ions are test particles, they would respond to the transverse electric field fluctuations $\mathbf{E}_{\perp}$ near the oxygen gyrofrequency locally according to the Langevin equation:

$$
d \mathbf{v}_{\perp} / d t=q_{i} \mathbf{E}_{\perp} / m_{i}
$$

To understand the stochastic nature of the Langevin equation, we visualize an ensemble of ions $f\left(\mathbf{v}_{\perp}\right)$ and study its stochastic properties. Assuming that the interaction times among the particles and the local electric field fluctuations are small compared to the global evolution time, we may write within the interaction time scale:

$$
f\left(\mathbf{v}_{\perp}, t+\Delta t\right)=\int f\left(\mathbf{v}_{\perp}-\Delta \mathbf{v}_{\perp}, t\right) P_{l}\left(\mathbf{v}_{\perp}-\Delta \mathbf{v}_{\perp}, \Delta \mathbf{v}_{\perp}\right) d \Delta \mathbf{v}_{\perp}
$$

where $P_{l}\left(\mathbf{v}_{\perp}-\Delta \mathbf{v}_{\perp}, \Delta \mathbf{v}_{\perp}\right)$ is the normalized transition probability of a particle whose velocity changes from $\mathbf{v}_{\perp}-\Delta \mathbf{v}_{\perp}$ to $\mathbf{v}_{\perp}$ in $\Delta t$, and $\Delta \mathbf{v}_{\perp}$ ranges over all possible magnitudes and transverse directions. Standard procedure at this point is to expand both sides of (13) in Taylor series expansions:

$$
\begin{aligned}
\frac{\partial f}{\partial t} \Delta t+O\left((\Delta t)^{2}\right) & =-\frac{\partial}{\partial \mathbf{v}_{\perp}} \cdot\left[\left\langle\Delta \mathbf{v}_{\perp}\right\rangle f\right] \\
& +\frac{1}{2} \frac{\partial^{2}}{\partial \mathbf{v}_{\perp} \partial \mathbf{v}_{\perp}}:\left[\left\langle\Delta \mathbf{v}_{\perp} \Delta \mathbf{v}_{\perp}\right\rangle f\right]+O\left(\left(\Delta \mathbf{v}_{\perp}\right)^{3}\right)
\end{aligned}
$$


where

$$
\begin{aligned}
& \left\langle\Delta \mathbf{v}_{\perp}\right\rangle=\int P_{l}\left(\mathbf{v}_{\perp}, \Delta \mathbf{v}_{\perp}\right) \Delta \mathbf{v}_{\perp} d\left(\Delta \mathbf{v}_{\perp}\right), \text { and } \\
& \left\langle\Delta \mathbf{v}_{\perp} \Delta \mathbf{v}_{\perp}\right\rangle=\int P_{l}\left(\mathbf{v}_{\perp}, \Delta \mathbf{v}_{\perp}\right) \Delta \mathbf{v}_{\perp} \Delta \mathbf{v}_{\perp} d\left(\Delta \mathbf{v}_{\perp}\right) .
\end{aligned}
$$

If we assume the $O\left(\left(\Delta \mathbf{v}_{\perp}\right)^{3}\right)$ terms are of order $(\Delta t)^{2}$ or higher, then in the limit of $\Delta t \rightarrow 0$, we obtain a Fokker-Planck equation, ${ }^{64,65}$ where the drift and diffusion coefficients are defined as: $\mathbf{D}_{1}=<\Delta \mathbf{v}_{\perp}>/ \Delta t$ and $\mathbf{D}_{2}=<\Delta \mathbf{v}_{\perp} \Delta \mathbf{v}_{\perp}>/ 2 \Delta t$ in the limit of $\Delta t \rightarrow 0$. These coefficients may be calculated straightforwardly using the Langevin equation. If the transition probability $P_{t}$ is symmetric in $\Delta \mathbf{v}_{\perp}$, then $\mathbf{D}_{1}$ vanishes and (14) leads to a diffusion equation in the transverse direction. We note that if the electric field fluctuations are Gaussian, then the higher order correlations of the fluctuations are automatically equal to zero.

We shall come back to the discussion of the effects of general intermittent fluctuations on particle energization processes. For the moment, let us assume the approach using the FokkerPlanck formulation is valid and proceed. Since we have assumed the time scale for the particlefluctuation interactions is much smaller than the global evolution time of the ion populations, we may then write the steady global evolution equation along an auroral field line $s$ under the guiding center approximation and neglecting the cross-field drift as: ${ }^{66-68}$

$$
\begin{array}{r}
\frac{\partial}{\partial s}\left[v_{\|} \frac{f}{B_{z}}\right]+\frac{\partial}{\partial v_{\|}}\left[-\frac{v_{\perp}^{2}}{2 B_{z}} \frac{d B_{z}}{d s} \frac{f}{B_{z}}\right]+\frac{1}{v_{\perp}} \frac{\partial}{\partial v_{\perp}}\left[v_{\perp} \frac{v_{\perp} v_{\|}}{2 B_{z}} \frac{d B_{z}}{d s} \frac{f}{B_{z}}\right] \\
=\frac{1}{v_{\perp}} \frac{\partial}{\partial v_{\perp}}\left[v_{\perp} D_{\perp} \frac{\partial}{\partial v_{\perp}} \frac{f}{B_{z}}\right]
\end{array}
$$

where $v_{\|}, v_{\perp}$ are the parallel and perpendicular components of the particle velocity with respect to the field-aligned direction. This expression may be interpreted as a convective-diffusion equation 
for the density of the guiding center ions per unit length of flux tube $f / B_{z}$, in the coordinate space of $\left(s, v_{\|}, v_{\perp}\right)$.

To evaluate $D_{1}$, the gyrotropic perpendicular diffusion coefficient, from $\mathbf{D}_{2}$, we recognize that the electric field fluctuations are broadband both in $k_{\perp}$ and $\omega$. Therefore, at all times, some portion of the fluctuations will be in resonance with the ions. The resonance condition, however, is strongly dependent on the localization and scale dependency of the intermittent fluctuations." We demonstrate below, as a simple illustrative example, how such resonant interactions may be accomplished by neglecting the Doppler shifts due to $\mathbf{k}$ such that only the intermittent fluctuations clustering around the instantaneous gyrofrequency of the ions provide the main contributions to the diffusion process. Standard arguments then lead to the following expression for the perpendicular diffusion coefficient:

$$
D_{\perp}=\left(\pi q_{i}^{2} / 2 m_{i}^{2}\right)\left\langle\left|E^{2}\right|\left(\Omega_{i}\right)\right\rangle_{r}
$$

where $<\left|E^{2}\right|\left(\Omega_{i}\right)>_{r}$ is the resonant portion of the average of the square of the transverse electric field fluctuations evaluated at the instantaneous gyrofrequency of the ions, $\Omega_{i}$.

Measurements by polar orbiting satellites indicate that the electric field spectral density $\Sigma$ follows an approximate power law $\Sigma^{-\alpha}$ in the range of the local oxygen gyrofrequencies, where $\alpha$ is a constant. If we make the additional approximations by assuming that the spectrum observed at the satellite is applicable to all altitudes and choosing the geomagnetic field to scale with the altitude as $s^{-3}$, we would then expect $\Sigma\left(\Omega_{i}, s\right)$ to vary with altitude $s$ as $s^{3 \alpha}$. Because we have made some rather restrictive resonance requirements for the fluctuations to interact with the ions, we expect the resonant portion of the average of the square of the transverse electric field fluctuations to be only a fraction $\eta$ of the total measured electric field spectral density. Therefore, we arrive at the following approximate expression for the diffusion coefficient: 


$$
D_{\perp}=\left(\eta \pi q_{i}^{2} / 2 m_{i}^{2}\right) \Sigma_{0}\left(s / s_{o}\right)^{3 \alpha}
$$

We have performed global Monte Carlo simulations for Eqs. (16) and (18) for the conic event discussed by Retterer et al. ${ }^{67}$ with $\alpha=1.7$ and $\Sigma_{0}=1.9 \times 10^{-7}(\mathrm{~V} / \mathrm{m})^{2} \mathrm{sec} / \mathrm{rad}$. Top panel of Figure 9 shows the measured oxygen velocity distribution contours and the second panel of Fig. 9 shows the corresponding calculated contours for $\eta=1 / 8$ at the satellite altitude of $s_{o}=2 R_{E}$. Thus, with one eighth of the measured electric field spectral density contributing, the broadband electric field fluctuations can adequately generate an oxygen distribution function with the energy and shape comparable to that obtained from observations. We have also calculated the oxygen ion distributions for a range of altitudes under the same conditions. Figure 10 is a plot of the average parallel energy versus the average perpendicular energy per oxygen ion as the ions evolve upward along the geomagnetic field line. We note that as the energies increase with altitudes, the ratio of the energies becomes nearly a constant.

These results are comparable to our previous calculated results based on the assumption that the relevant fluctuations were purely field-aligned propagating electromagnetic ion cyclotron waves. $^{66-68}$ As discussed in the previous sections, we generally expect the coexistence of nonpropagating transverse electrostatic nonlinear fluctuations and a small fraction of field-aligned propagating waves in the auroral zone. Thus, the ion energization process in the auroral zone is probably due to a combination of both types of fluctuations. As it has been discussed in Chang et $a l^{66}$, an asymptotic solution exhibiting such behavior may be obtained analytically in closed form. Therefore, in the asymptotic limit (i.e, at sufficiently high altitudes), it is expected that such an ion distribution will become entirely independent of its low altitude initial conditions. In fact, it has been shown by Crew and Chang ${ }^{68}$ that the ion distributions will become self-similar at sufficiently high altitudes and everything will scale with the altitude.

The above sample calculations did not include the self-consistent electric field that must be determined in conjunction with the energization of the ions as well as the electrons. This is 
particularly relevant in the downward auroral current region where the electric field can provide a significant pressure cooker effect such as that suggested by Gorney et al. ${ }^{69}$ and demonstrated convincingly by Jasperse ${ }^{70}$ and Jasperse and Grossbard ${ }^{71}$ based on global evolutional calculations similar to those considered by Tam and Chang ${ }^{72-75}$ for the solar wind and Tam et al. ${ }^{76,77}$ for the polar wind. These ideas will not be considered in this treatise.

We now return to the discussion of the effect of intermittency on ion heating. Measurements of the electric field spectral density are generally limited by the response capabilities of the measuring instruments. The faster the instruments can collect data, the more refined the scales of the measurements. As it has been seen in Sec. IV, we expect the measured spectrum density to exhibit small-scale intermittency behavior. In fact, it is known that fast response measurements generaily exhibit strongiy intermittent signatures of the fluctuations. In the diffusion approximation, the ion energization process is limited by the amplitude of the second moment of the probability distribution of the fluctuations. This amplitude may become smaller as the scale of measurements is reduced. Thus, in the limit of small scales, the amplitude of the measured spectrum may decrease and thereby requiring a larger value of $\eta$ to accomplish the same level of energization.

But, the effects of the intermittency of the fluctuations on particle energization may be underestimated if we stay within the diffusion approximation. As it can be seen from the derivation of the diffusion approximation above, only the second order correlations of the fluctuations were included in the energization process. Since for intermittent turbulence, the probability distributions of the fluctuations are generally non-Gaussian, the effects of the intermittency can manifest in the higher order correlations beyond the second order diffusion coefficient. This implies that the higher order correlations of the velocity fluctuations may be of the order of $\Delta t$ and therefore cannot be neglected in Eq. (14). Under such circumstances, the Fokker-Planck and diffusion approximations of the ion energization processes can become 
inadequate. A more appropriate approach to address such non-Gaussian stochastic processes is to refer directly to the functional equation (13) using the non-Gaussian transition probability or the Langevin equation (12) with the actual intermittent time series of the electric field fluctuations.

We have performed global simulations based on Eq. (13) for non-Gaussian intermittent fluctuations exhibiting the shape suggested by Castaing et $a l^{58}$ :

$$
\Pi_{\lambda}(\xi)=\frac{1}{2 \pi \lambda} \int_{0}^{\infty} \exp \left(-\frac{\xi^{2}}{2 \sigma^{2}}\right) \exp \left(-\frac{\ln ^{2}\left(\sigma / \sigma_{0}\right)}{2 \lambda^{2}}\right) \frac{\mathrm{d} \sigma}{\sigma^{2}}
$$

where $\xi$ represents either the $x$ - or $y$-component of the dimensionless transverse velocity fluctuations and $\lambda>0$ is a parameter that characterizes the intermittency. We set $\ln \sigma_{0}=-\lambda^{2}$, to ensure the variance equal to unity. For $\lambda=0$, Eq. (19) reduces to a Gaussian distribution. As $\lambda$ increases, the degree of intermittency increases. Third panel of Fig. 9 shows the contours calculated for $\lambda=1$ with $\eta=1 / 8$. For this case, the degree of intermittency is not strong enough to significantly affect the value of $\eta$. But with strong intermittent fluctuations $(\lambda=2$, bottom panel of Fig. 9), a value of $\eta$ equal to $1 / 5$ is required to adequately generate the ion conic to observed energies.

\section{SUMMARY}

We have provided a modern description of dynamical complexity relevant to the intermittent turbulence of coexisting nonpropagating spatiotemporal fluctuations and propagating modes in space plasmas. The theory is based on the physical concepts of sporadic and localized interactions of coherent structures that emerge naturally from plasma resonances.

The technique of the dynamic renormalization group is applied to the study of forced and/or self-organized criticality (FSOC), scale invariance, and symmetry breaking, related to such type of multiscale fluctuations. We also demonstrated that the particle interactions with the intermittent turbulence could lead to the efficient energization of the plasma populations such as auroral ions. Numerical examples are presented to illustrate the concepts and methodology. 


\section{ACKNOWLEDGMENTS}

This exposition touches upon a broad range of research areas covering the physics of space plasmas and complexity. The authors wish to thank their past and present colleagues, M. André, V. Angelopoulos, R. Bruno, S. Chandrasekhar, S.C. Chapman, G. Consolini, B. Coppi, G.B. Crew, G. Ganguli, M. Goldstein, A. Hankey, J.R. Jasperse, S. Jurac, C.F. Kennel, P. Kintner, M. Kivelson, A. Klimas, A.T.Y. Lui, E. Marsch, W. Matthaeus, P. De Michelis, J.F. Nicoll, J.M. Retterer, C. Seyler, A.S. Sharma, M.I. Sitnov, H.E. Stanley, D. Tetreault, V. Uritsky, J. Valdivia, D. Vassiliadis, D. Vvedensky, N. Watkins, J. Weygand, F. Yasseen, and J.E. Young for very useful discussions. Our wavelet analysis employed some of the wavelet software provided by C. Torrence and G. Compo, which is available at URL: http://paos.colorado.edu/research/wavelets. This research was partiaily supported by AFOSR, NASA and NSF. 


\section{References}

${ }^{\prime}$ T. Chang, in AGU Monograph on "Encounter between Global Observations and Models in the ISTP Era“, edited by J.L.Horwitz, D.L. Gallagher, and W.K. Peterson (American Geophysical Union, Washington, D.C., 1998), Vol. 104, p.193.

${ }^{2}$ T. Chang, Proceedings $4^{\text {th }}$ International Conference on Substorms, edited by Y. Kamide et al. (Kluwer Academic Publishers, Dordrecht, The Netherlands, and Terra Scientific Publishing Company, Tokyo, 1998), p. 431.

${ }^{3}$ T. Chang, in Astrophysics and Space Science, edited by J. Büchner et al. (Kluwer Academic Publishers, Dordrecht, The Netherlands, 1998), Vol. 264, p. 303.

${ }^{4}$ T. Chang, Phys. Plasmas 6, 4137 (1999).

'T. Chang, in Solar Wind Ten, edited by M. Velli, R. Bruno, and F. Malara (AIP Conference Proceedings, Melville, NY, 2003), Vol.679, p. 481.

${ }^{6}$ T. Chang, S.W.Y. Tam, C.C. Wu, and G. Consolini, Space Science Reviews 107, 425 (2003).

${ }^{7}$ C.C. Wu and T. Chang, Geophys. Res. Lett. 27, 863 (2000).

${ }^{8}$ C.C. Wu and T. Chang, IEEE Trans. on Plasma Science 28, 1938 (2000).

${ }^{9}$ C.C. Wu and T. Chang, Journal of Atmospheric Sciences and Terrestrial Physics 63, 1447 (2001).

${ }^{10}$ T. Chang, D.D. Vvedensky, and J.F. Nicoll, Physics Reports 217, 279 (1992).

${ }^{11}$ T. Chang, Physica Scripta T89, 80 (2001).

${ }^{12}$ B.V. Waddell, B. Carreras, H.R. Hicks, and J.A. Holmes, Phys. Fluids 22, 896 (1979).

${ }^{13}$ D. Tetreault, J. Geophys. Res. 97, 8531 (1992).

${ }^{14}$ J.B. Taylor, Phys. Rev. Lett. 33, 1139 (1974).

${ }^{15}$ P.H. Rutherford, Phys. Fluids 16, 1903 (1973).

${ }^{16}$ D. Tetreault, J. Geophys. Res. 97, 8541 (1992). 
${ }^{17}$ (a) R. Bruno, V. Carbone, L. Sorriso-Valvo, B. Bavassano, J. Geophys. Res. 108(A3), 10.1029/2002JA009615 (2003). (b) R. Bruno, V. Carbone, P. Veltri, E. Pietropaolo, and B. Bavassano, Planetary and Space Science 49, 1201 (2001).

${ }^{18}$ R. Bruno, V. Carbone, L. Sorriso-Valvo, and B. Bavassano, EOS Trans. AGU, 83(47), Fall Meeting Suppl., Abstract SH12A-0396 (2002).

${ }^{19}$ T. Chang, C.C. Wu, and V. Angelopoulos, Physica Scripta T98, 48 (2002).

${ }^{20}$ C.E. Seyler, Jr., Geophys. Res. Lett. 15, 756 (1988).

${ }^{21}$ W.H. Matthaeus, M.L. Goldstein, and D.A. Roberts, J. Geophys. Res. 95, 20673 (1990).

${ }^{22}$ H.R. Strauss, Phys. Fluids 19, 134 (1976).

${ }^{23}$ D. Biskamp, Nonlinear Magnetohydrodynamics (Cambridge University Press, Cambridge, U.K., 1993).

${ }^{24}$ G. Consolini and T. Chang, Space Science Reviews 95, 309 (2001).

${ }^{25}$ T. Chang, IEEE Trans. on Plasma Science 20, 691 (1992).

${ }^{26}$ T. Chang, J.F. Nicoll, and J.E. Young, Physics Letters 67A, 287 (1978).

${ }^{27}$ K.G. Wilson and J. Kogut, Physics Reports 12C, 76 (1974).

${ }^{28}$ P. Bak, C. Tang, and K. Wiesenfeld, Phys. Rev. Lett. 59, 381 (1987).

${ }^{29}$ H.E. Stanley, Introduction to Phase Transitions and Critical Phenomena (Oxford University Press, New York, 1971).

${ }^{30}$ E.T. Lu, Phys. Rev. Lett. 74, 2511 (1995); E.T. Lu and R.J. Hamilton, Astrophysical J. 380, L89 (1991).

${ }^{31}$ G. Consolini, in Cosmic Physics in the Year 2000, edited by S. Aiello, N. Lucci, G. Sironi, A. Treves and U. Villante (Soc. Ital. di Fis., Bologna, Italy, 1997), p. 123.

${ }^{32}$ A.T.Y. Lui, S.C. Chapman, K. Liou, P.T. Newell, C.I. Meng, M. Brittnacher, and G.D. Parks, Geophys. Res. Lett. 27, 911 (2000).

${ }^{33}$ V.M. Uritsky, A.J. Klimas, D. Vassiliadis, D. Chua, and G.D. Parks, J. Geophys. Res. 107, 1426 (2002). 
${ }^{34}$ V. Angelopoulos, T. Mukai, and S. Kokubun, Phys. Plasmas 6, 4161 (1999).

${ }^{35}$ (a) G. Boffetta, V. Carbone, P. Giuliani, P. Veltri, and A. Vulpiani, Phys. Rev. Lett. 83, 4662 (1999). (b) M.P. Freeman, N.W. Watkins, and D.J. Riley, Geophys. Res. Lett. 27, 1087 (2000). (c) N.W. Watkins, Nonlinear Processes in Geophysics 9, 389 (2002).

${ }^{36}$ D. Baker, A.J. Klimas, R.L. McPherron, and J. Büchner, Geophys. Res. Lett, 17, 41 (1990).

${ }^{37}$ A.J. Klimas, D.N. Baker, D.A. Roberts, D.H. Fairfield, and J. Büchner, J. Geophys. Res. 97, 12253 (1992).

${ }^{38}$ A.S. Sharma, D. Vassiliadis, and K. Papadopoulos, Geophys. Res. Lett. 20, 335 (1993).

${ }^{39}$ T. Chang and C.C. Wu, Phys. Plasmas 9, 3679 (2002).

${ }^{40}$ A.J. Klimas, J.A. Valdivia, D. Vassiliadis, D.N. Baker, M. Hesse, and J. Takalo, J. Geophys. Res.. 105, 18765 (2000).

${ }^{41}$ W.H. Matthaeus and M.L. Goldstein, Phys. Rev. Lett. 57, 495 (1986).

${ }^{42}$ M.I. Sitnov, A.S. Sharma, K. Papadopoulos, D. Vassiliadis, J.A. Valdivia, A.J. Klimas, and D.N. Baker, J. Geophys. Res. 105, 12955 (2000).

${ }^{43}$ S.C. Chapman, N.W. Watkins, R.O. Dendy, P. Helander, and G. Rowlands, Geophys. Res. Lett. 25, 2397 (1998).

${ }^{44}$ N.W. Watkins, S.C. Chapman, R.O. Dendy, and G. Rowlands, Geophys. Res. Lett. 26, 2617 (1999).

${ }^{45}$ A.T.Y. Lui, in Physics of Space Plasmas, edited by T. Chang and J.R. Jasperse (MIT Geo/Cosmo Plasma Physics, Cambridge, MA, 1998), Vol. 15, p. 233.

${ }^{46}$ M. Hoshino, A. Nishida, T. Yamamoto, and S. Kokubum, Geophys. Res. Lett. 21, 2935 (1994).

${ }^{47}$ A. Milovanov, L. Zelenyi, and G. Zimbardo, J. Geophys. Res. 101, 19903 (1996).

${ }^{48} \mathrm{M}$. André and T. Chang, in Physics of Space Plasmas, edited by T. Chang and J.R. Jasperse (Scientific Publishers, Inc., Cambridge, MA, 1992), Vol. 12, p. 35.

${ }^{49}$ C.Y. Tu and E. Marsch, Space Science Reviews 73, 1 (1995).

${ }^{50}$ T. Chang and H.E. Stanley, Phys. Rev. B8, 1178 (1973). 
${ }^{51}$ T. Chang, A. Hankey, and H.E. Stanley, Phys. Rev. B7, 4263 (1973).

${ }^{52}$ T. Chang, A. Hankey, and H.E. Stanley, Phys. Rev. B8, 346 (1973).

${ }^{53}$ J.F. Nicoll, T. Chang, and H.E. Stanley, Phys. Rev. Lett. 32, 1446 (1974).

${ }^{54}$ J.F. Nicoll, T. Chang, and H.E. Stanley, Phys. Rev. Lett. 36, 113 (1976).

${ }^{55}$ B. Hnat, S.C. Chapman, G. Rowlands, N.W. Watkins, and W.M. Farrel, Geophys. Res. Lett. 29(10), 10.1029/2001GL014587 (2002).

${ }^{56}$ L. Sorriso-Valvo, V. Carbone, P. Veltri, G. Consolini, and R. Bruno, Geophys. Res. Lett. 26, 1801 (1999).

${ }^{57}$ (a)M. Forman and L.F. Burlaga, in Solar Wind Ten, edited by M. Velli, R. Bruno, and F. Malara (AIP Conference Proceedings, Melville, NY, 2003), Vol. 679, p. 554. (b) J. Weygand (private communication, 2003).

${ }^{58}$ B. Castaing, Y. Cagne, and E.J. Hopfinger, Physica D 46, 177 (1990).

${ }^{59} \mathrm{~S}$. Jurac (private communication, 2003).

${ }^{60}$ M. Farge, Annual Reviews of Fluid Mechanics 24, 395 (1992).

${ }^{61}$ C. Meneveau, J. Fluid Mech. 232, 469 (1991).

${ }^{62}$ G. Consolini, T. Chang, and A.T.Y. Lui, "Complexity and topological disorder in the Earth's magnetotail dynamics," in Non-equilibrium Transition in Plasmas, edited by A.S. Sharma and P. Kaw (Kluwer Academic Publishers, Dordrecht, The Netherlands), to be published.

${ }^{63}$ P.J. Morrison and R.D. Hazeltine, Phys. Fluids 27, 886 (1984).

${ }^{64}$ A. Einstein, Ann. d. Physik 17, 549 (1905).

${ }^{65}$ S. Chandrasekhar, Rev. Mod. Phys. 15, 1 (1943).

${ }^{66}$ T. Chang, G.B. Crew, N. Hershkowitz, J.R. Jasperse, J.M. Retterer, and J.D. Winningham, Geophys. Res. Lett. 13, 636 (1986).

${ }^{67}$ J.M. Retterer, T. Chang, G.B. Crew, J.R. Jasperse, and J.D. Winningham, Phys. Rev. Lett. 59, 148 (1987).

${ }^{68}$ G.B. Crew and T. Chang, Phys. Fluids 31, 3425 (1988). 
${ }^{69}$ D.J. Gorney, Y.T. Chiu, and D.R. Croley, J. Geophys. Res. 90, 4205 (1985).

${ }^{70}$ J.R. Jasperse, Geophys. Res. Lett. 25, 3485 (1998).

${ }^{71}$ J.R. Jasperse and N.J. Grossbard, IEEE Trans. Plasma Science 28, 1874 (2000).

${ }^{72}$ S.W.Y. Tam and T. Chang, Geophys. Res. Lett. 26, 3189 (1999).

${ }^{73}$ S.W.Y. Tam and T. Chang, Comments on Modern Physics, Vol. 1, Part C, 141 (1999).

${ }^{74}$ S.W.Y. Tam and T. Chang, Geophys. Res. Lett. 28, 11351 (2001).

${ }^{75}$ S.W.Y. Tam and T. Chang, Astronomy \& Astrophysics 395, 1001 (2002).

${ }^{76}$ S.W.Y. Tam, F. Yasseen, T. Chang, and S.B. Ganguli, Geophys. Res. Lett. 22, 2107 (1995).

${ }^{77}$ S.W.Y. Tam, F. Yasseen, and T. Chang, Ann. Geophys. 16, 948 (1998). 


\section{Figure Captions}

1. Field-aligned spatiotemporal coherent structures.

2. Cross-sectional view of coherent structures of the same polarity. Contours indicate constants of magnetic flux and arrows indicate directions of magnetic field. Blackened area is an intense current sheet (strong magnetic shear).

3. 2D MHD simulations of interacting coherent structures. Left panels are contours of magnetic flux at $t=300$. Corresponding current density distributions are given in the right panels. For reference, the sound wave and Alfvén wave traveling times through a distance $2 \pi$ are about 4.4 and 60 , respectively.

4. Renormalization-group trajectories and fixed points.

5. Fourier spectra of $B^{2}(k)$ at $t=300$ (solid curves) and 600 (dashed curves) for the 2D MHD simulations with $(512 \times 512)$ and $(1024 \times 1024)$ grid points. The straight lines have slopes of -2 . Note that the scaling range is more extended for the larger simulation.

6. 2D MHD simulation results with $(512 \times 512)$ grid points for the probability density distributions of the difference of the square of the magnetic field fluctuations at scales of $\delta=$ 2 (red), 4 (green), 8 (black), 16 (blue) and 32 (magenta) units of grid spacing. Left panels display the calculated results of $P\left(\delta B^{2}, \delta\right)$ and the right panels are the corresponding scaled distributions.

7. Wavelet analyses of the $2 \mathrm{D}$ MHD simulation results with $(512 \times 512)$ grid points. Top panels show raw spatial distributions of $J_{z}$ or $B^{2}$. Bottom left panel of (1) gives the power spectrum of complex Morlet wavelet transform of the current density $J_{z}$ and right bottom panel of (1) gives the contours of the corresponding $\operatorname{logLIM}(1)$ of $B^{2}$ for the homogeneous case. Left bottom panel of (2) gives the contours of $\operatorname{logLIM}(1)$ of $B^{2}$ at the neutral sheet 
region and right bottom panel gives the corresponding contours away from the neutral sheet region for the shear case. The $x$-axis and scales are in units of the grid points.

8. LIM(2) of $B^{2}$ using the Morlet wavelet transform for (a) the homogeneous case and (b) shear case $(+1+$ for $y=1.5 \pi$ and $\circ \circ \circ$ for $y=1.8 \pi)$.

9. Observed and calculated velocity contour plots for conic event of Retterer et al. ${ }^{67}$ Top panel: observed contours. Second panel: Simulation results based on the diffusion approximation $(\lambda=0)$. Third panel: Simulation results for weak intermittency $(\lambda=1)$. Bottom panel: Simulation results for strong intermittency $(\lambda=2)$.

10. Solid line depicts $W_{\|}$versus $W_{\perp}$ for simulated conic events. Dashed line is the asymptote predicted by Chang et al. ${ }^{66}$ 
Fig. 1

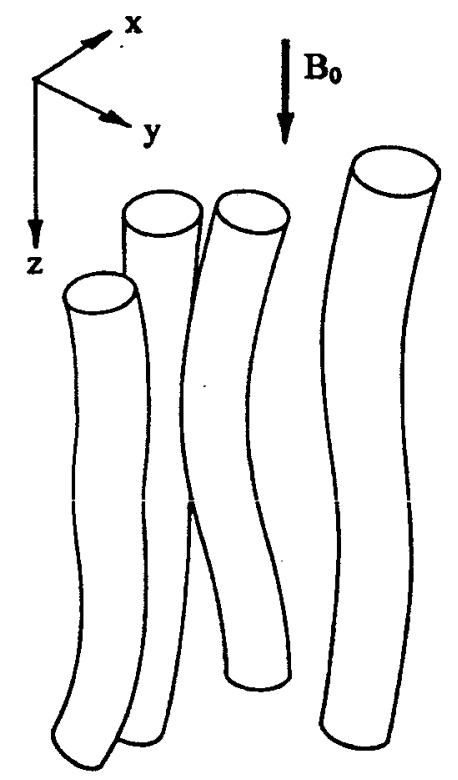


Fig. 2

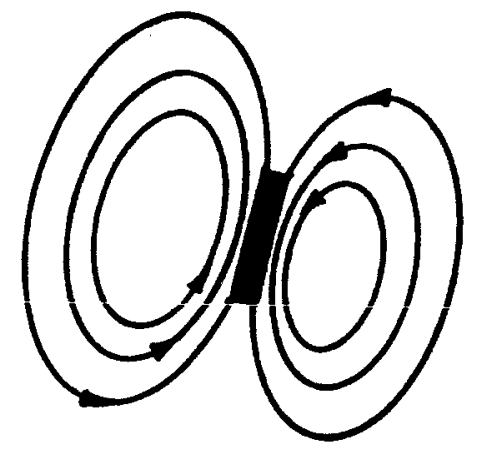


Fig. 3

(a) Homogeneous Case $(512 \times 512)$
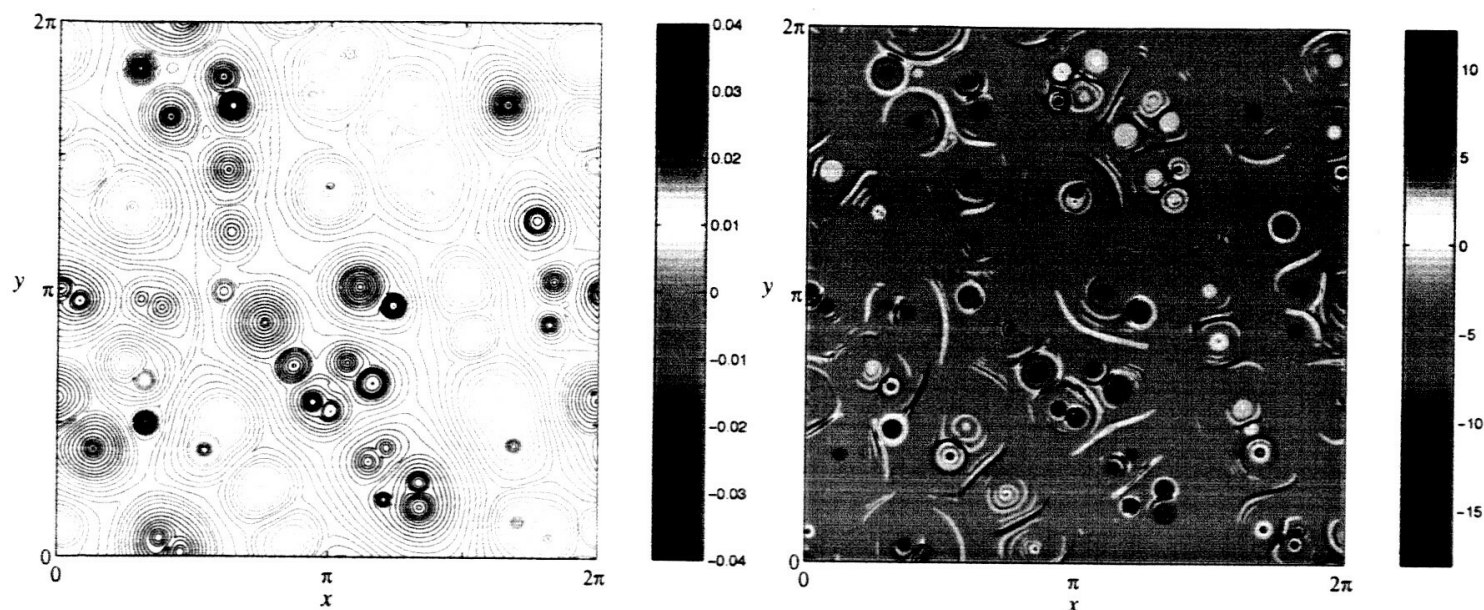

(b) Homogeneous Case $(1024 \times 1024)$
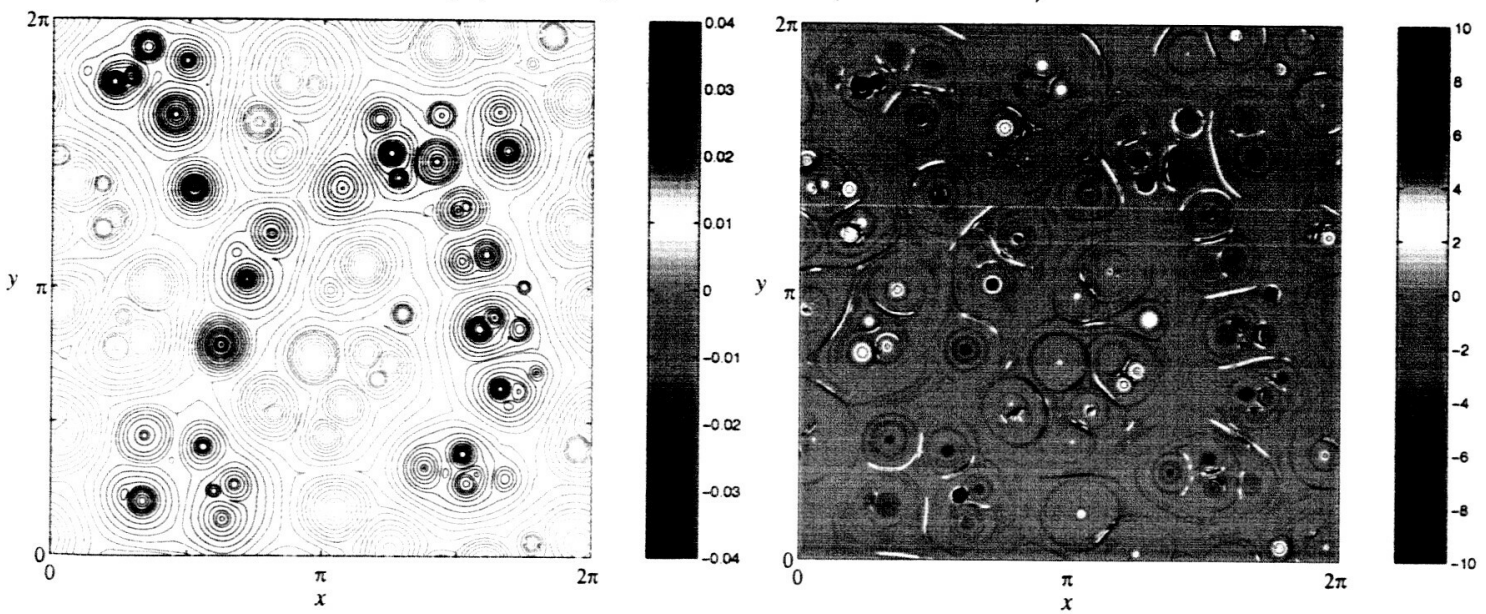

(c) Shear Case $(512 \times 512)$
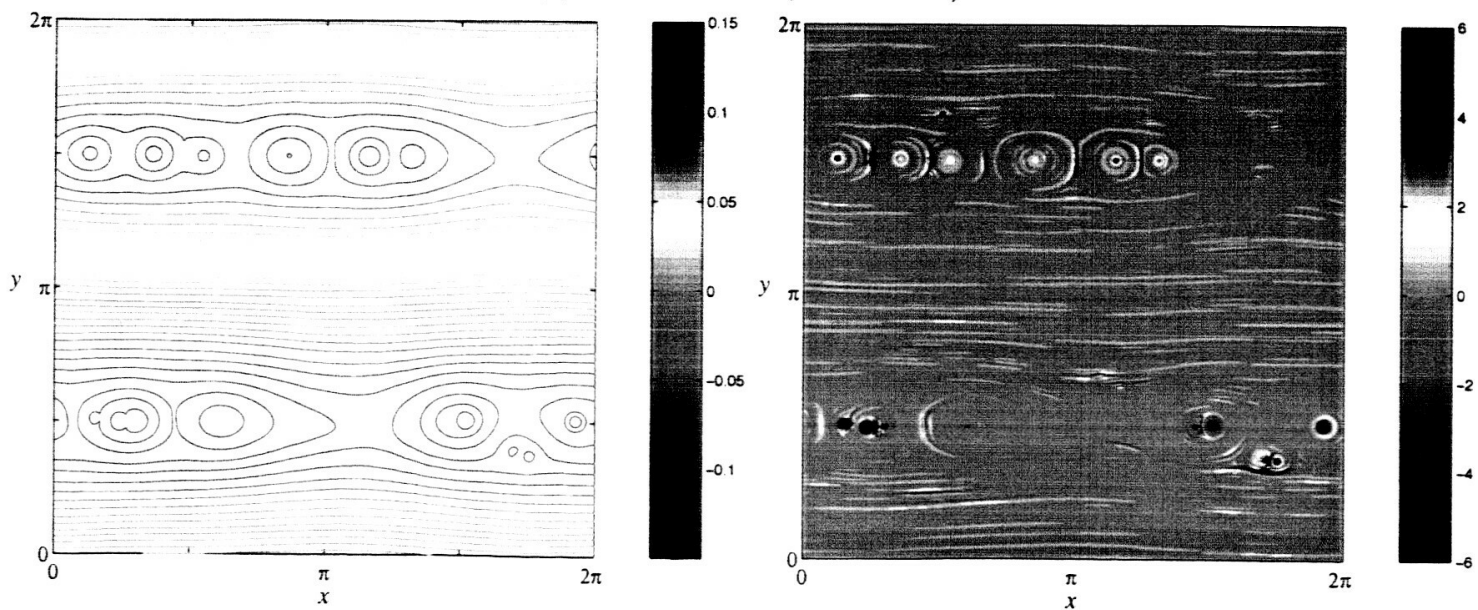
Fig. 4

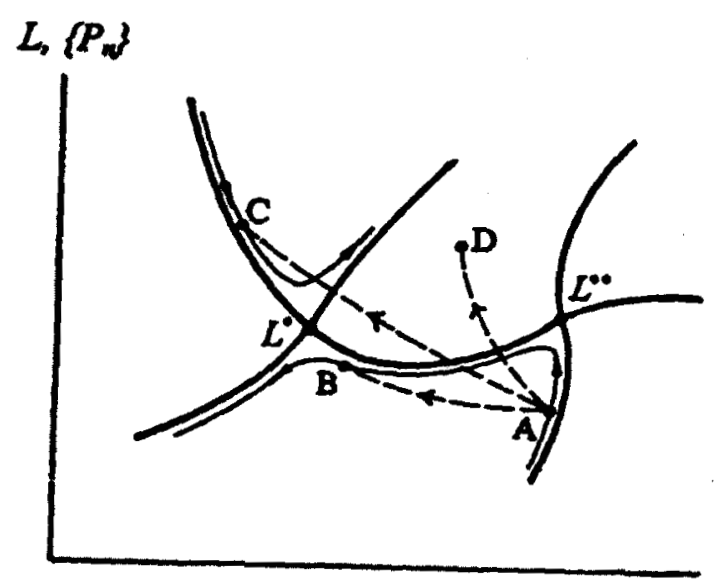


Fig. 5
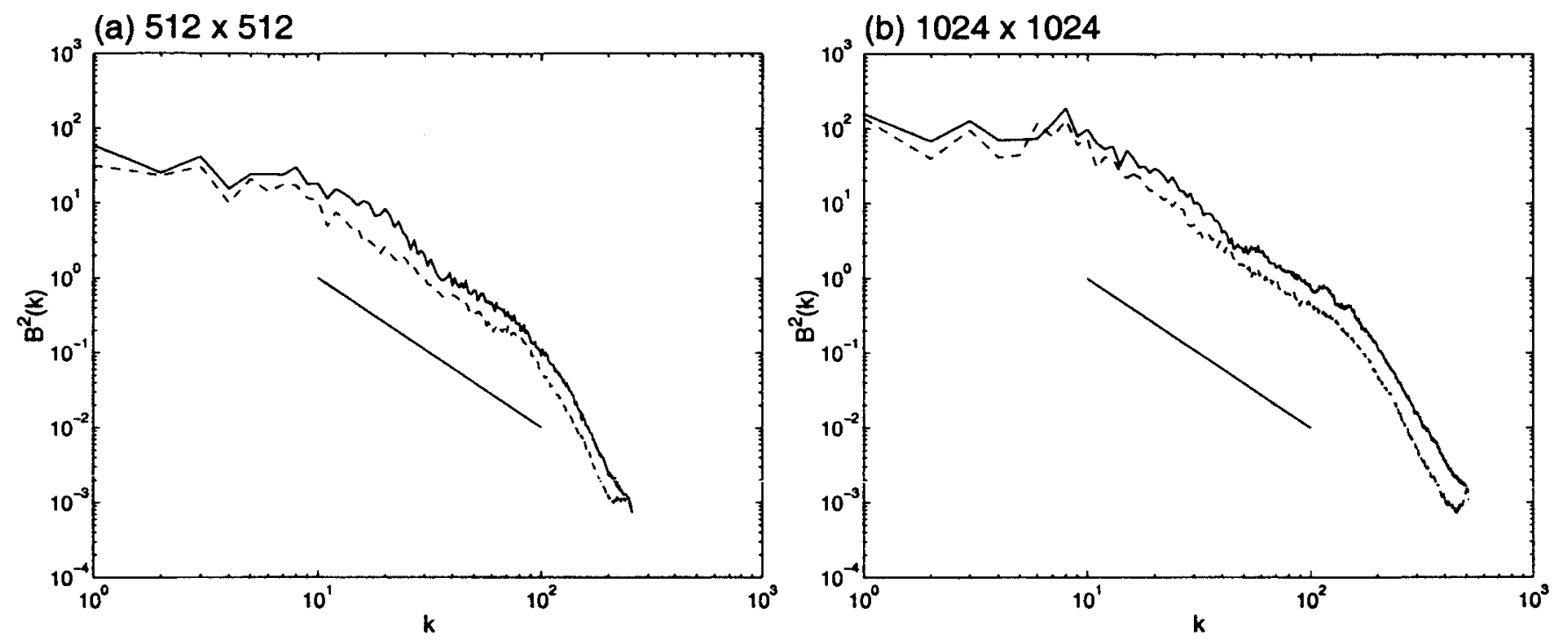
Fig. 6

(a) Homogeneous Case
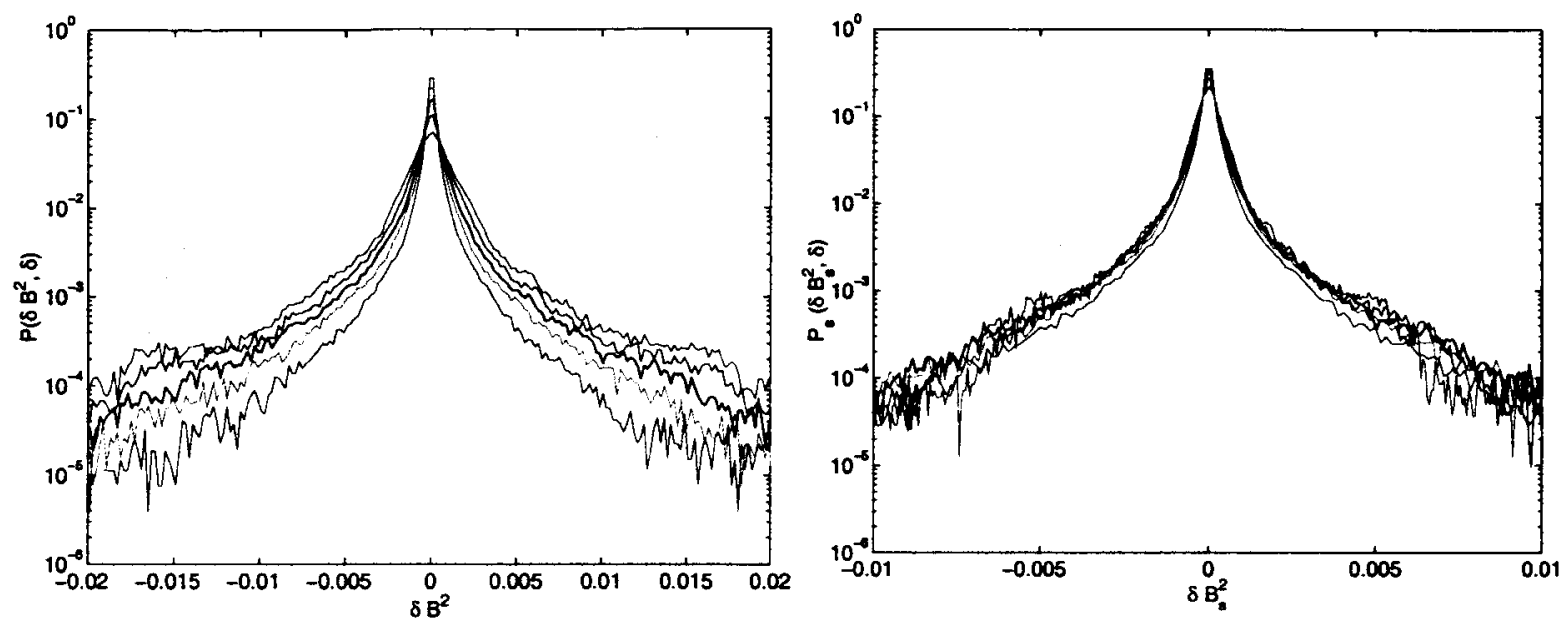

(b) Shear Case - Near the Neutral Sheet
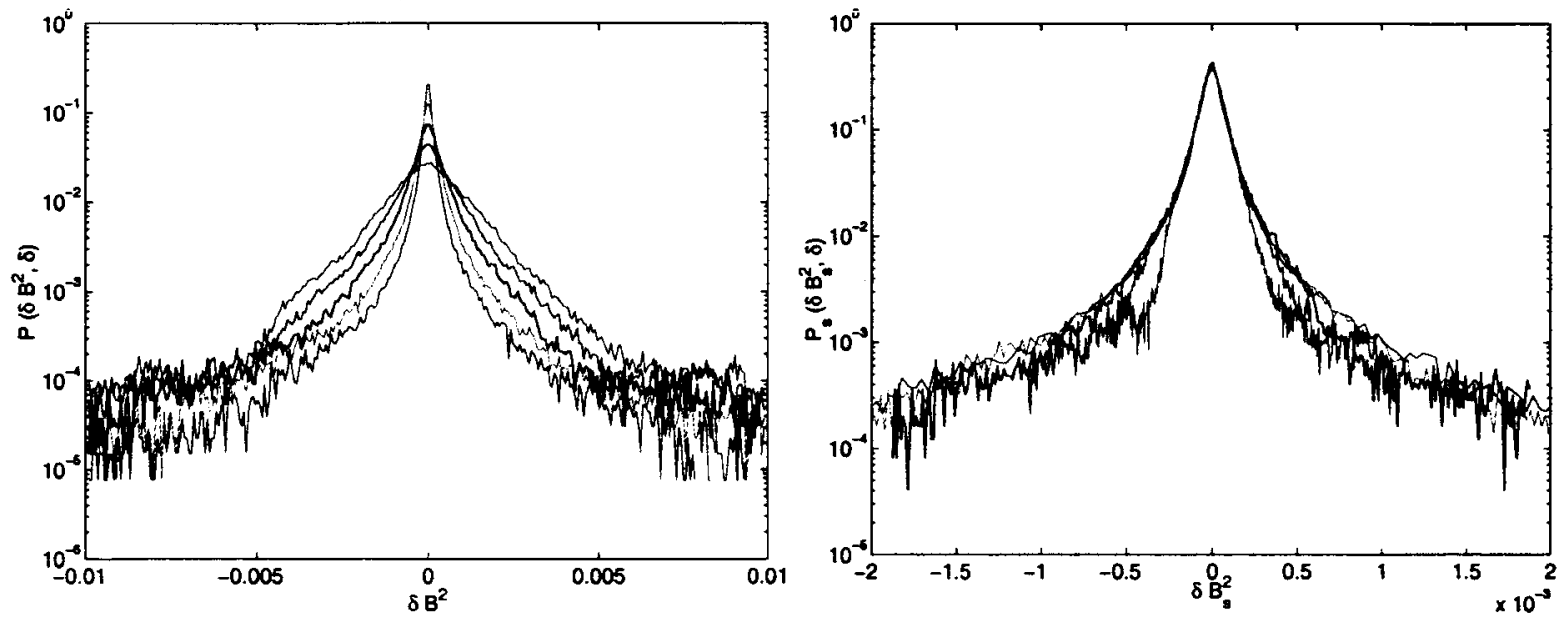

(c) Shear Case - Away from the Neutral Sheet
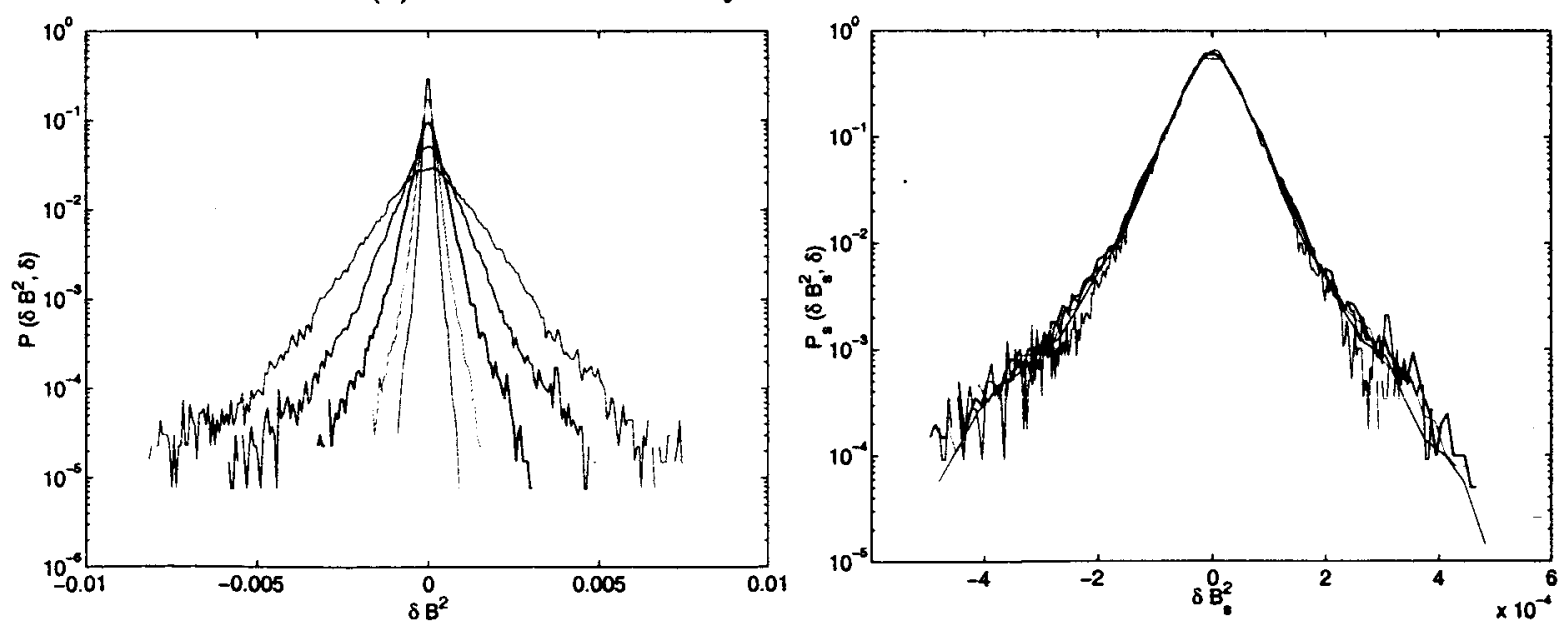
Fig. 7

(1) Homogeneous Case

a) $J_{z}$ along $y=\pi$

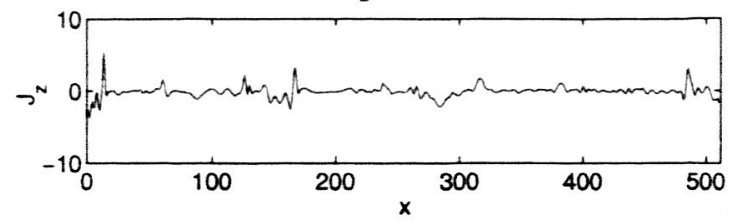

b) $\mathrm{J}_{\mathbf{z}}$ Wavelet Power Spectrum (Log Scale)

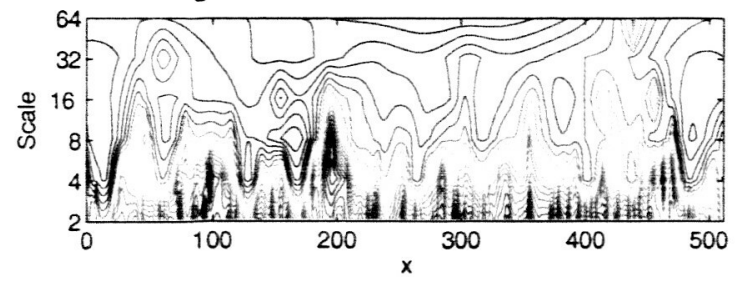

a) $B^{2}$ along $y=\pi$

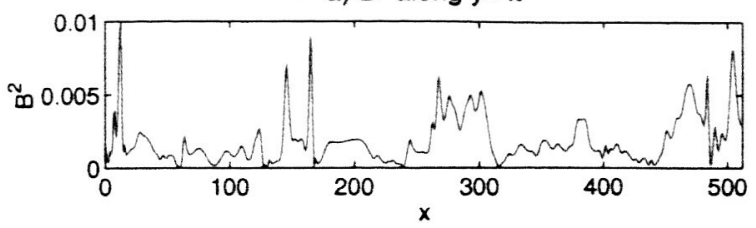

b) $B^{2}$ Wavelet LogLIM(1)
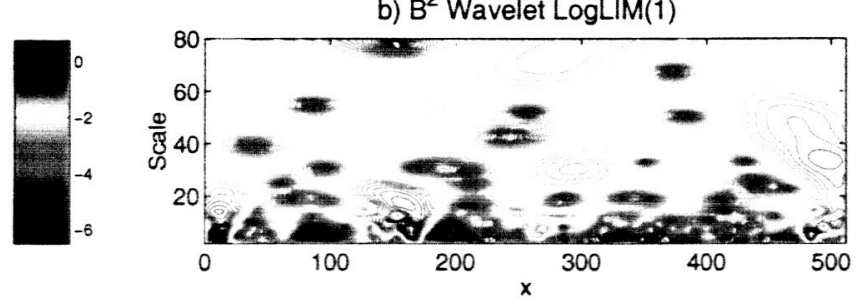

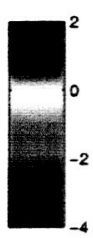

(2) Shear Case

a) $B^{2}$ along $y=1.5 \pi$

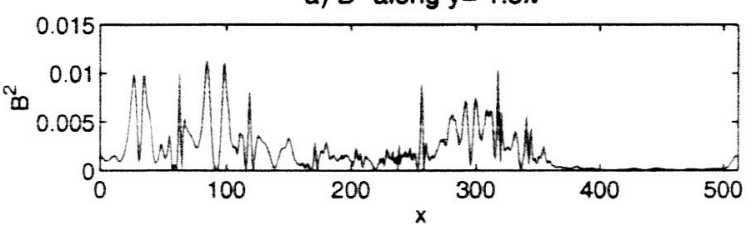

b) $B^{2}$ Wavelet LogLIM(1)

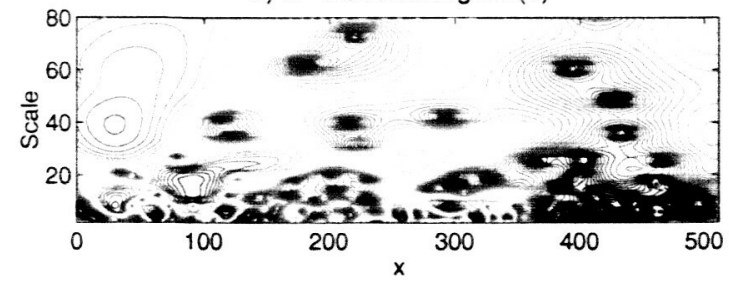

a) $B^{2}$ along $y=1.8 \pi$

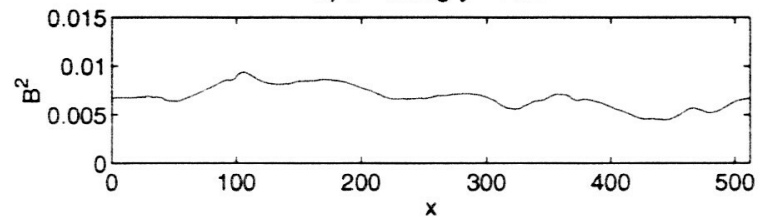

b) $B^{2}$ Wavelet LogLIM(1)
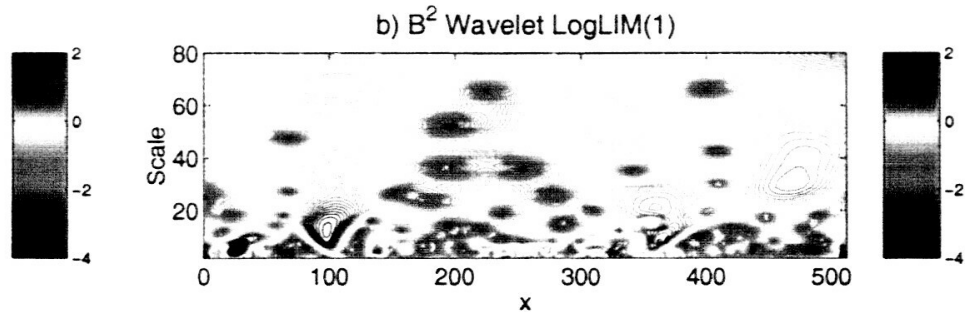
Fig. 8

(a) Homogeneous Case

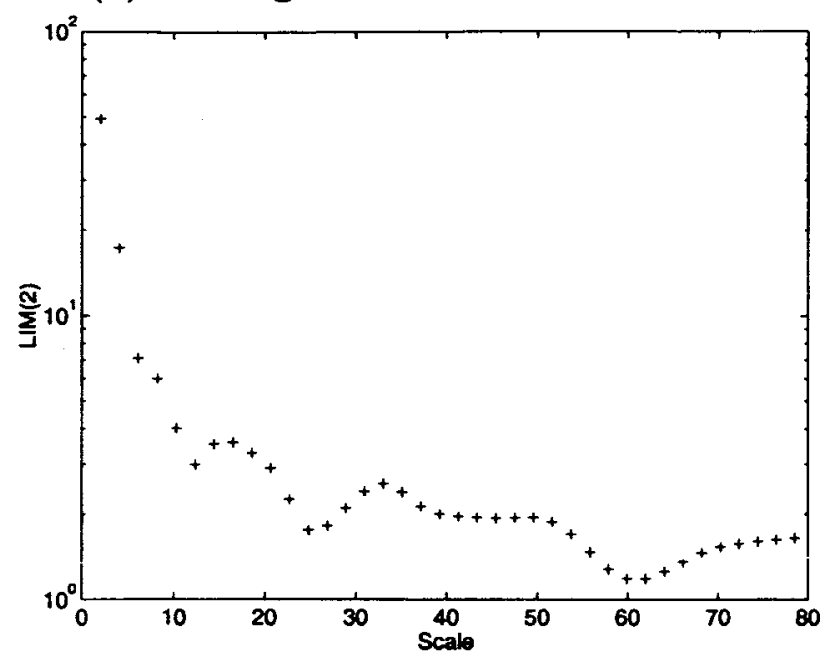

(b) Shear Case

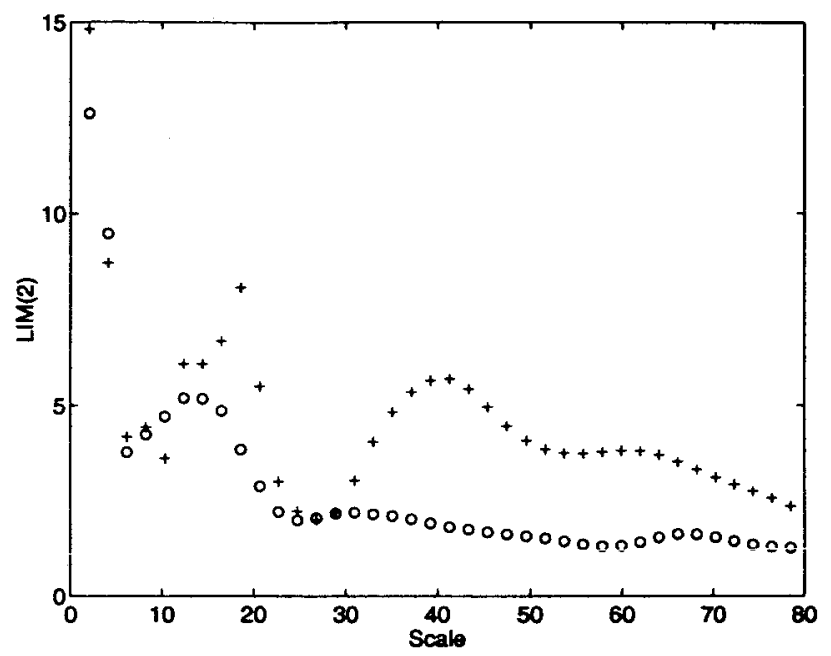


Fig. 9
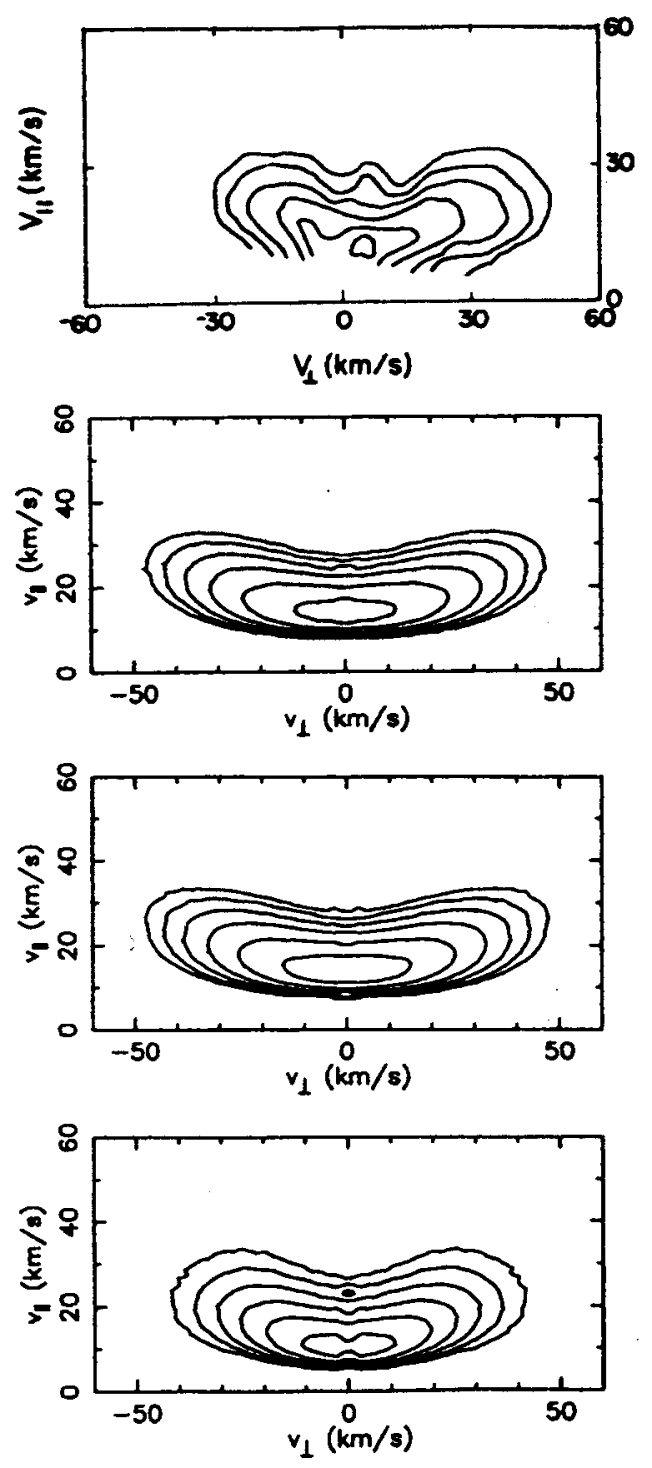
Fig. 10

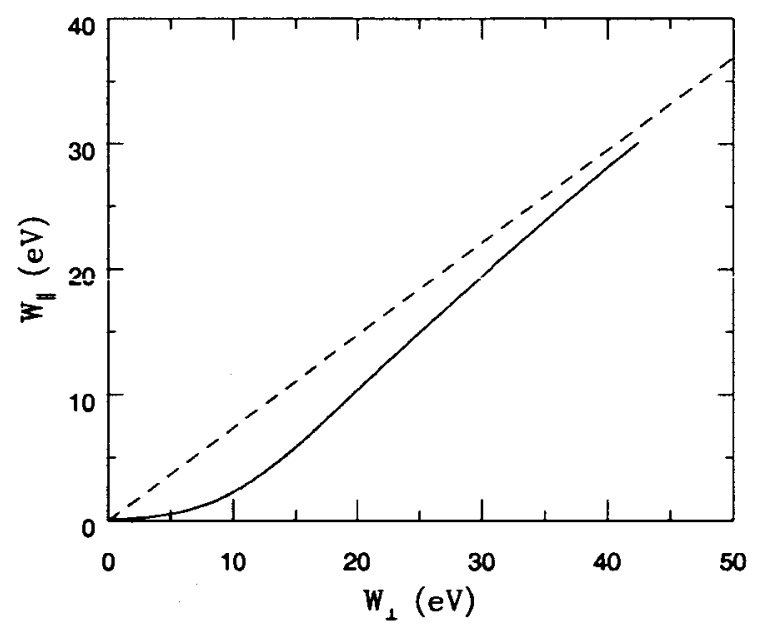

